\title{
Optimal Allocation of Shunt Dynamic Var Source SVC and STATCOM: A Survey
}

\author{
Wenjuan Zhang, Student Member, IEEE, Fangxing Li, Senior Member, IEEE, Leon M. Tolbert, Senior Member, IEEE
}

\begin{abstract}
Optimal allocation of Var source involves optimal location and determination of the size of the Var source. The purpose is to find the right tradeoff between the investment cost of the new Var source and the benefits in system operation derived from the presence of the additional reactive compensators. Traditionally, the locations for placing new Var sources were either simply estimated or directly assumed. This paper categorizes the literature relevant to optimal allocation of shunt dynamic Var source SVC and STATCOM, based on the voltage stability analysis tools used. Those tools include static voltage stability analysis ones such as $\mathrm{P}-\mathrm{V}$ and $\mathrm{V}-\mathrm{Q}$ curve analysis, continuation power flow (CPF), optimization methods (OPF), modal analysis, saddle-node bifurcation analysis, and dynamic voltage stability analysis ones such as Hopf bifurcation analysis and time-domain simulation. Static voltage stability analysis techniques are also used in dynamic Var planning. At the end of the paper, the advantages of static and dynamic voltage stability analysis tools are summarized.
\end{abstract}

Index Terms-reactive power planning (RPP), reactive power optimal allocation, voltage stability analysis, CPF, modal analysis, OPF, SVC, STATCOM

\section{INTRODUCTION}

Recently network blackouts related to voltage collapse tend to occur from lack of reactive power support in heavily stressed conditions, which are usually triggered by system faults. Calvaer [1] stated that a system may undergo a voltage collapse if it includes at least one voltage collapse bus. Chebbo et al. [2] noted that the cause of the 1977 New York blackout was proved to have been a reactive power problem, and the 1987 Tokyo blackout was believed to have been due to a reactive power shortage and a voltage collapse during a summer peak load. However, reactive power has received less attention recently until the Great Blackout in August 2003 in the northeastern US, which showed that reactive power in US power systems was not very well planned and managed [3]. Reactive power including its planning process has received tremendous interest after the 2003 Blackout from utilities, independent system operators (ISOs), researchers, and the government.

Power electronics based equipment, or Flexible AC Transmission Systems (FACTS), provide proven technical solutions to voltage stability problems. Especially, due to the increasing need for fast response for power quality and voltage stability, the shunt dynamic Var compensators such

W. Zhang, F. Li, and L. M. Tolbert are with the Department of Electrical and Computer Engineering, The University of Tennessee, Knoxville, TN 37996-2100 USA (e-mail: wzhang5@utk.edu, fli6@utk.edu, tolbert@utk.edu). This work was supported in part by the National Science Foundation under Contract NSF ECS-0093884 and Oak Ridge National Laboratory under Contract 4000041689. as Static Var Compensators (SVC) and Static Synchronous Compensators (STATCOM) have become feasible alternatives to a fixed reactive source, and therefore have received intensive interests. There are more than 50 SVCs installed in the United States, ranging from 30 MVar to 650 MVar each [4]. STATCOMs are installed at several sites in the United States, ranging between 30 MVar and 100 MVar each [5]-[8].

FACTS make the application of a large amount of Var compensation more efficient, flexible, and attractive. Consequently, a series of questions have been raised frequently by utility planners and manufacturers: where is the right location and what is the right size for the installation of reactive power compensators considering technical and economic needs? Can the models, methods, and tools used for static Var planning be applied in dynamic Var planning? The answers to these questions are needed for utilities to make better use of these new power electronic controlled Var sources.

In order to answer the above questions, it should be stated that optimal allocation of static and dynamic Var sources belongs to the Reactive Power Planning (RPP) or Var planning category. RPP deals with the decision on new Var source location and size to cover normal, as well as, contingency conditions. The planning process aims at providing the system with efficient Var compensation to enable the system to be operated under a correct balance between security and economic concerns.

Essentially, RPP is a large-scale nonlinear optimization. The solution techniques of Optimal Power Flow (OPF) have evolved over many years, each with its particular mathematical and computational characteristics [9]. The majority of the techniques discussed in the literature of the last 20 years use at least one of the following 3 categories of methods.

- Conventional methods (Local optimum): A group of methods such as Generalized Reduced Gradient (GRG), Newton's Approach, and Successive Quadratic Programming (SQP) for NLP problem are often trapped by a local optimal solution.

- Heuristic methods (Near-global optimum): In recent years, the intelligent optimization techniques such as Simulated Annealing (SA), Genetic Algorithms (GA), and Tabu Search (TS) have received widespread attention as possible techniques to get a global optimum for RPP problem, but these methods are time consuming. 
- Sensitivity based methods: index, modal or eigenvalue analysis.

This paper is organized as follows. Section II illustrates the Voltage Stability Analysis (VSA) tools such as Continuous Power Flow (CPF), modal analysis, and OPF. Section III presents SVC planning literature review. Section IV presents the STATCOM planning literature review. Section V concludes the paper.

\section{VOLTAGE STABILITY ANALYSIS TOOLS}

Voltage collapse studies, an integral part of VSA of power systems, are of growing importance for the design and operation of power systems. The main function of shunt reactive power compensation is for voltage support to avoid voltage collapse. Then, voltage stability is a very important consideration when the location and size of new Var sources need to be determined during Var planning. Many analytical methodologies have been proposed and are currently used for the study of this problem: static voltage stability analysis tools such as P-V and V-Q curve analysis, Continuation Power Flow, optimization methods, modal analysis, saddlenode bifurcation analysis, and dynamic voltage stability analysis tools such as Hopf bifurcation analysis, time-domain simulation. Static voltage stability analysis techniques are also used in dynamic Var planning. All these techniques in the literature will be explained in this section.

\section{A. Static Voltage Stability Analysis Techniques}

Static voltage stability analysis is concerned with two main aspects:

- Determination of how far the system is operating from the voltage collapse point using $\mathrm{CPF}$ based on bifurcation theory, or OPF considering a given load increase pattern and generator sharing scheme.

- Identification of buses or areas prone to voltage instability problems by using modal analysis

\section{1) Continuation Power Flow (CPF)}

The Point of Collapse (PoC) is given by the nose point of $\mathrm{P}-\mathrm{V}$ or $\mathrm{V}-\mathrm{Q}$ curve, where the voltage drops rapidly with an increase in load demand as shown in Fig. 1. PoC is also known as the equilibrium point, where the corresponding Jacobian becomes singular, power flow solution fails to converge beyond this limit, which is indicative of voltage instability, and can be associated with a saddle-node bifurcation point.

Of the different types of bifurcations, saddle-node bifurcations are of particular interest in power systems, because they have been shown as one of the primary causes for "static" voltage collapse problems [10]. These instabilities are usually local area voltage problems due to the lack of reactive power, and hence by increasing the static voltage stability margin (SM) defined as the distance between the saddle-node-bifurcation point and the base case operating point as shown in Fig. 1, one could expect an improvement on the stability of the system for that operating point. Hence, determining the location of this point is of practical importance in power systems.

CPF was originally developed to determine bifurcation points of Ordinary Differential Equations (ODE) systems, and have been successfully applied to the computation of collapse points in power systems [11][12][13]. CPF was first developed to overcome the ill-conditioning near the critical point, where the Jacobian matrix of the Newton-Raphson method becomes singular.

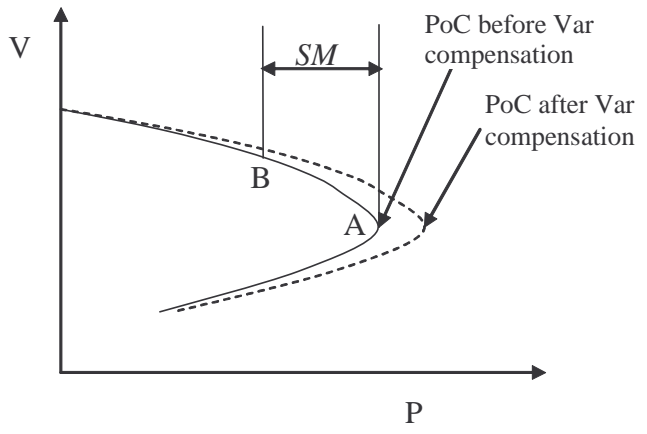

Fig. 1. P-V curve

\section{2) Optimal Power Flow (OPF)}

Most of the numerical tools such as continuation methods used in voltage collapse studies are based on concepts and/or techniques developed from bifurcation analysis of power systems. More recently, however, new optimization-based tools have been developed to study voltage collapse problems in power systems. It is demonstrated in [14] that bifurcation theory, is basically equivalent to some typical optimization methodologies. A voltage collapse point computation problem can be formulated as an optimization problem, known as Total Transfer Capability (TTC).

It is obvious that there are similarities and strong ties between tools developed for the computation of collapse points from bifurcation theory and those based on optimization techniques. Several computational methods based on bifurcation theory have been shown to be efficient tools for VSA; however, it is difficult to introduce operational limits and computationally expensive to use continuation method, especially for large systems with multiple limits. Using optimization techniques for these types of studies present several advantages, especially due to their limit handling capabilities. Further extension has been made to include the security (contingency) constraints in the OPF model, which is known as SCOPF model.

The majority of the Var planning objective is to provide the least cost of Var source with feasible voltage magnitudes as constraints. The Optimal Power Flow (OPF) model fits the need of Var planning naturally and can be written as follows:

$$
\begin{aligned}
& \operatorname{Min} \sum_{i \in N c}\left[C_{0 i}+C_{1 i} \times Q_{c i}\right] \times r_{i} \\
& \text { subject to } \\
& P_{g i}-P_{L i}-P(V, \theta)=0 \text { (active power balance) } \\
& Q_{g i}+Q_{C i}-Q_{L i}-Q(V, \theta)=0 \text { (reactive power balance) } \\
& \left|L F_{k}\right| \leq L F_{k}^{\max } \text { (line flow limits) }
\end{aligned}
$$




$$
\begin{aligned}
& V_{i}^{\min } \leq V_{i} \leq V_{i}^{\max } \quad \text { (bus voltage limits) } \\
& P_{g i}^{\min } \leq P_{g i} \leq P_{g i}^{\max } \quad \text { (active power limits) } \\
& Q_{g i}^{\min } \leq Q_{g i} \leq Q_{g i}^{\max } \quad \text { (reactive power limits) } \\
& Q_{c i}^{\min } \leq Q_{c i} \leq Q_{c i}^{\max } \quad \text { (Var source limits) } \\
& \text { where } C_{0 i}-\text { the fixed Var source installation cost } \\
& C_{I i}-\text { the per unit Var source purchase cost } \\
& Q_{c i}-\text { Var source installed at bus } i \\
& N_{C}-\text { set of possible Var source installment buses } \\
& r_{i}-1 \text { if there is installation of reactive power source at } \\
& \text { bus } i, \text { otherwise, it is zero } \\
& P_{g i}-\text { generator active power output } \\
& P_{L i}-\text { load active power } \\
& Q_{g i}-\text { generator reactive power output } \\
& Q_{L i}-\text { load reactive power } \\
& L F_{k} \text { - transmission line flow } \\
& V_{i}-\text { bus voltage }
\end{aligned}
$$

\section{3) Modal Analysis}

Modal or eigenvalue analysis of the system Jacobian ( $\mathbf{J}$ ) matrix of the system load flow equation, near the point of voltage collapse, which is obtained at the point of maximum power transfer capability of the system, can be used to identify buses vulnerable to voltage collapse and locations where injections of reactive power benefit the system most.

The participation of each load in the critical mode (near PoC) determines the importance of the load in the collapse. The degree of participation is determined from an inspection of the entries of the left eigenvector of the critical mode. The components of the left eigenvector can be interpreted as indicating a direction normal to the operational boundary of the system. Right eigenvector components indicate the degree to which given variables are involved in a given mode. The use of both left and right eigenvector information leads to the notion of participation factors. The participation factors indicate which generators should be motivated to inject more active or reactive power into the system, and where the load shedding would be more effective to increase the stability margin.

\section{B. Dynamic Voltage Stability Analysis Techniques}

\section{1) Hopf Bifurcation Point}

Not all events of voltage collapse in power systems can be associated to saddle-node bifurcations, as other bifurcations have also been shown to induce collapse, such as Hopf bifurcations which corresponds to dynamic voltage stability [15]. Therefore, voltage instabilities directly related to Hopf bifurcations have been categorized as dynamic voltage collapse problems. The Hopf bifurcation occurs when the pair of complex eigenvalues of Jacobian lies exactly on the imaginary axis when the parameter is slowly varied.

\section{2) Time-Domain Simulation}

The conventional transient stability time domain simulation programs have been greatly enhanced over recent years to make them suitable for assessment of long-term and voltage stability problems. However, time domain simulation is usually combined with static voltage stability analysis tools such as modal analysis in dynamic Var planning.

\section{SVC PLANNING}

\section{A. Priority Based Algorithm}

\section{1) CPF and Modal Analysis}

Traditional voltage stability analysis tools such as continuation power flow (CPF) for PoC or modal analysis at $\mathrm{PoC}$ to determine weak areas or buses are used in [16] for SVC location. The SVC size is determined based on the need to continuously meet the voltage stability margin requirement. The speed with which the shunt device should operate is a separate issue. The dynamic aspects of the voltage collapse phenomenon cannot be properly analyzed by modal analysis based on power flow static models.

$\mathrm{CPF}$ and modal analysis are also used to indicate the candidate buses for voltage support in [17]. However, the modal analysis is helpless regarding the minimum amount of reactive support to solve the voltage collapse problem. As a result, OPF is used to determine the MVar rating of the reactive compensator, $\mathrm{SVC}$ is still described as voltage independent Var source $Q_{c}$ like in static Var planning, but in fact it is voltage dependent Var source.

The location of a SVC device is determined by the participation factors from modal analysis and the use of controllability indices of the most critical stability state in the modal analysis in [18]. It evaluates the extended steady-state voltage stability margins in electric power system due to FACTS controller such as SVC and UPFC.

In [19], the dynamic voltage stability margin has been considered as the distance between the Hopf bifurcation point and the base case operating point, the static voltage stability margin as shown if Fig. 1. SVC should be placed at a bus, which produces maximum enhancement in both margins. The saddle-node bifurcation points have been obtained by a continuation power flow software package UWPFLOW. A method has been proposed to combine the two margins in [19], which computes static and dynamic participation factors and thereafter, combined hybrid participation factors at different buses. The bus with maximum value of the hybrid participation factor for the intact system and critical contingency cases has been selected as the candidate bus for the SVC placement. This paper is one of the few papers which consider not only static margin but dynamic margin.

\section{2) Loss Sensitivity Index}

The SVC has been considered from a static point of view to reduce the total system real power transmission loss $\left(P_{L}\right)$ in [28]. Loss sensitivity index is proposed as the sensitivity of total transmission loss with respect to the control parameters of SVC for their optimal placement. The bus with the most negative loss sensitivity value is preferred to be the SVC location. SVC has been considered as a reactive power source with the following reactive power limits: 


$$
\begin{gathered}
Q_{S V C}=V_{t}\left(V_{t}-V_{r e f}\right) / X_{s l} \\
Q_{\text {ind }}=B_{\text {ind }} V^{2}{ }_{\text {ref }} \\
Q_{\text {cap }}=B_{\text {cap }} V_{r e f}^{2}
\end{gathered}
$$

where $X_{s l}$ is the equivalent slope reactance in p.u. equal to the slope of voltage control characteristics, and $V_{t}$ and $V_{\text {ref }}$ are the node and the reference voltage magnitudes, respectively. $Q_{S V C}$ is valid as long as it is within inductive limits $Q_{\text {ind }}$ and capacitive limits $Q_{c a p}$ set by available inductive and capacitive susceptances ( $B_{\text {ind }}$ and $B_{\text {cap }}$, respectively).

After deciding the optimal location of SVC, the formulation of OPF to minimize real power losses as the objective has been developed to determine the size. The same methodology is used in [29], but the OPF objective minimizes fuel cost. The method based on Loss Sensitivity Index ignores the voltage stability limits, and only considers the economical aspect.

\section{B. Optimization Based Algorithm}

\section{1) Maximize voltage stability margin}

From the viewpoint of optimal reactive reinforcement for voltage stability, optimal SVC planning is evaluated in [20] such that the reactive margin (Q-margin) of the study configuration including critical modes is maximized by using a hybrid method based on the simulated annealing (SA) and Lagrange multiplier techniques. The system reactive margin is defined as the maximum amount of extra reactive demand that the system can supply, before it reaches a critical point and encounters a voltage instability problem.

The optimal SVC planning is treated as a multi-objective optimization in [21] for maximizing the system reactive power margin, minimizing system real power losses and voltage depressions at critical points. Fuzzy logic techniques are applied to transform the multi-objective optimization problem into a constrained problem with a single objective function known as the fuzzy performance index. SVC is still modeled as voltage independent $\operatorname{Var}$ source $Q_{c}$.

\section{2) Minimize Voltage Deviation}

A two-stage Tabu Search (TS) is proposed in [22] for determining the location and the output of SVCs devices to reduce the voltage deviation in distribution system with DG. The location is expressed as a discrete variable, and the rating of SVC is a continuous one. SVC is treated as voltage independent Var source $Q_{c}$ in the model. However, postcontingency state security and voltage stability margin are ignored in this paper.

\section{3) Minimize Cost}

The objective of the optimal allocation of SVC in [23] is to minimize the overall cost function, which includes the investment costs of FACTS and the bid offers of the market participants. The cost function of SVC is as follows [24]:

$$
\mathrm{C}(s)=0.0003 s^{2}-0.3051 s+127.38(\mathrm{US} \$ / \mathrm{kVar})
$$

where $\mathrm{C}(s)$ is in US\$/kVar and $s$ is the operating range of the FACTS devices in MVar. The proposed approach converts the objective into minimizing the overall cost function consisting of FACTS like SVC devices investment cost and fuel cost in [25]. Genetic Algorithm (GA) is used in both papers [23] [25]; however, post-contingency state security and voltage stability margin are still ignored.

The location of SVC for reactive compensation is chosen according to reactive marginal cost criterion in [26], whose value can be obtained from the OPF solution corresponding to the Lagrange multiplier of the reactive power constraint. The objective is the $\$$ improvement due to the reduction of the fuel cost in this paper. Thus, for each bus in the system, there will be a corresponding reactive marginal cost. The bus with the highest reactive marginal cost will be chosen as the SVC location.

In most of the work, the placement of FACTS controllers has been considered for the intact system normal operating state. Very limited efforts have been made to study the impact of these controllers and their placement under contingencies. Therefore, if contingency and voltage stability are considered, it may be a considerable improvement in this field.

In [27], the objective is to minimize the sum of the new FACTS investment costs such as $F=\left(C_{0}+C_{l} Q_{c}\right) \cdot x$, corrective control cost such as fast load shedding cost, and preventive control cost to improve the voltage stability margin in all predefined contingencies. Meanwhile, the bus voltage profile and voltage stability margin are kept within specified limits in normal and the corresponding contingency states. Two sets of constraints including normal condition constraints and critical mode constraints are formulated in the OPF. The problem is formulated as a large-scale Mixed Integer Nonlinear Programming (MINLP), which is solved by a two level hybrid GA/SLP method. However, the shunt FACTS model is still a voltage independent Var source $Q_{c}$ in the OPF.

\section{STATCOM PLANNING}

\section{A. $C P F$ and Modal Analysis}

The main goal of the voltage stability study in [30] is to keep the static voltage Stability Margin $(S M)$ based on active power (P-margin) greater than at least $5 \%$ at $\mathrm{N}-1$ contingency states, usually such a margin is treated as a safe one, which is defined as:

$$
S M=\frac{\sum_{i} P_{i}^{\text {critical }}-\sum_{i} P_{i}^{\text {normal }}}{\sum_{i} P_{i}^{\text {normal }}}
$$

where $P_{i}^{\text {normal }}$ and $P_{i}^{\text {critical }}$ are the MW loads of load bus $i$ at normal operating state $\mathrm{B}$ and the voltage collapse critical state (PoC) A as shown in Fig. 1, respectively. This means that after a single branch outage, the power system can afford 5\% active load increment without voltage collapse occurring. For this purpose, the software package Interactive Power Flow Program (IPFLOW) is the main tool for steady state calculation, and then the Voltage Stability Analysis Program (VSTAB) by EPRI for PoC calculation and modal analysis is used to determine the best location for installing STATCOM as remedial measures against voltage collapse. VSTAB is 
also for contingency assessment to ensure the rating of STATCOM is enough to keep the $5 \%$ stability margin.

The optimal location of some FACTS devices including STATCOM in [31] is determined from the viewpoint of increasing the loadability margin of a power system by applying continuation power flow tool. Power System Analysis Toolbox (PSAT) software with power flow and continuation power flow functions is applied to calculate and compare the P-V curves with and without FACTS device. A detailed STATCOM power injection model is proposed to use in the continuation power flow as shown in Fig. 2. In the several buses with the same minimum loadability margin, the one with the weakest voltage profile is chosen as the STATCOM location. However, no contingency cases are involved in the analysis.

So far no work has been reported in open literature for the optimal location of STATCOM considering the effects on economical cost and voltage stability margin under both normal and contingency circumstances.

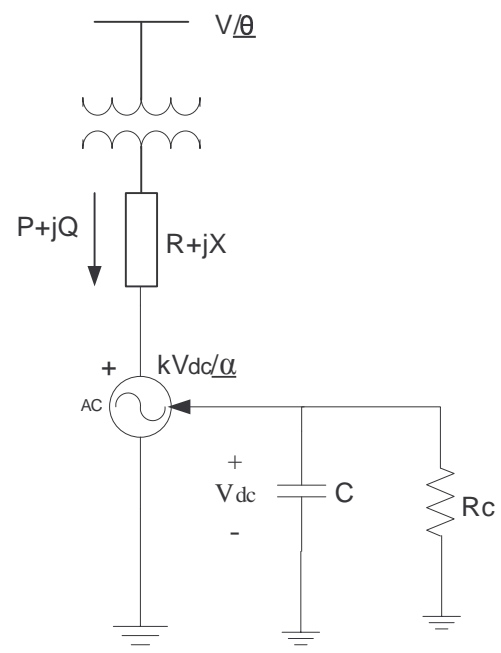

Fig. 2. STATCOM power injection model

\section{B. Time Domain Simulation vs. Static Voltage Stability MW Margin}

Reference [32] focuses on a STATCOM as a dynamic Var source providing voltage support in a power system. Both static voltage stability margins based on P-V curve and timedomain dynamic simulation are carried out and compared to verify the agreement between the two study methods. Even though one method is static analysis and the other is dynamic analysis, the two different methods lead to the same result. For example, voltage collapse in time-domain simulation reflects on $\mathrm{P}-\mathrm{V}$ curve as the operating point out of the range of maximum load capability.

\section{Time Domain Simulation vs. Modal Analysis}

Modal analysis and time-domain simulation are used to determine the best location for the STATCOM controllers in [33]. Three violations for voltage stability criteria are defined in this paper, which are recovery voltage less than $90 \%$ of its initial value; transient voltage less than $80 \%$ of its initial value; and oscillations remaining for more than 20 cycles.

First, critical contingencies that result in voltage unstable condition are identified by the sign of the eigenvalues computed in modal analysis. Secondly, time-domain simulation is performed to test all critical contingencies and identify the types of criteria violations for each critical contingency. By comparing the results from both modal analysis and dynamic analysis, a good correlation between the two techniques is found: buses with high participation factors in modal analysis are the same that have violations to the voltage stability criteria in dynamic analysis. Thirdly, the candidate locations of FACTS controllers are selected by combining the bus participation factor from modal analysis and the number of violations from the dynamic analysis. However, this testing method to decide the final size and location ignores the economic analysis. As a result, it is not accurately based on the maximum benefit.

In [34], the results for voltage stability analysis from the dynamic analysis using time domain simulations and the static analysis using modal analysis are compared, which are shown to be consistent in indicating system voltage stability.

\section{CONCLUSIONS}

Voltage stability is indeed a dynamic phenomenon and may be studied using a set of differential and algebraic equations. However the static approach is shown to have a number of practical advantages over the dynamic approach, which make the static approach more attractive.

- It requires only small modifications of a standard load flow program, so it is computationally less intensive.

- The P-V curves can cover a wide range of system operating conditions, whereas the time-domain simulations are for only one operating point. As a result, time-domain simulation usually requires a large number of study cases at different system operating conditions and contingencies.

- The P-V curves can provide much more information on the relationship between system and control parameters and voltage stability. An index value "voltage stability margin" is effectively used to illustrate the impact on the voltage stability for changing system parameters and Var source size parameters. Time-domain simulation can tell us the voltage profile of every bus, but can not demonstrate how far away to the voltage collapse point from the present operating point.

- Modal analysis can clearly indicate whether the system is stable or not at the given operating mode. In addition, the participation factors clearly define areas prone to voltage instability and indicate elements which are important to improve the system voltage stability most effectively.

Advantage of dynamic analysis is as follows:

- Time-domain simulation can clearly show the transient process and how long it will take to transfer to another stable operating point, which would not be illustrated in the P-V curve. The static voltage stability margin in the $\mathrm{P}-\mathrm{V}$ curve can not guarantee transient stability. 
- It is necessary to use dynamic analysis when to decide how fast the Var source needs to respond to the contingencies, thereafter, to decide the type of Var source such as dynamic Var source or static Var source.

The response of system voltage to a disturbance and system behavior during a voltage collapse situation can be considered as dynamic power system phenomena. However as far as reactive long term planning is concerned, a steadystate analysis has been shown to be generally adequate for providing an indicator of the margin from current operating point to voltage collapse point and for determining the location and MVar rating of any necessary reactive power source. Although dynamic analysis is separately used to design the controls for system reactive support, the advantages of the above static analysis make it suitable for the Var planning under a large number of conditions. In the end, the technologies in the literature of SVC and STATCOM planning are summarized in Table1. It should be noted that some methods that are originally designed for one compensator may be applied to the other with slight modification due to the similarity of SVC and STATCOM. If detailed models of SVC or STATCOM are desired, the specific features of the compensators need to be incorporated in the Var planning method, as shown in some previous works.

Table 1. Dynamic Var source planning technology.

\begin{tabular}{|c|c|c|}
\hline Technology & SVC(12) & STATCOM(4) \\
\hline CPF+ Modal analysis (no CA) & {$[16][17][18]$} & $\begin{array}{l}\text { [30] IPFLOW, } \\
\text { VSTAB }\end{array}$ \\
\hline CPF+ P-V curve (no CA) & & [31] PSAT \\
\hline $\begin{array}{l}\text { P-V curve + time-domain dynamic } \\
\text { simulation (CA) }\end{array}$ & & [32] \\
\hline $\begin{array}{l}\text { Modal analysis+ time-domain } \\
\text { dynamic simulation (CA) }\end{array}$ & & [33] \\
\hline Saddle-node \&Hopf bifurcation (CA) & [19] & \\
\hline Loss sensitivity index (no CA) & [28][29] & \\
\hline Max Q-margin (NLP)(SA)(no CA) & {$[20][21]$} & \\
\hline $\begin{array}{l}\text { Min voltage deviation } \\
(\mathrm{NLP})(\mathrm{TA})(\text { no } \mathrm{CA})\end{array}$ & [22] & \\
\hline $\begin{array}{l}\text { Min Var cost and bid offers (SA)( no } \\
\text { CA) }\end{array}$ & [23] & \\
\hline $\begin{array}{l}\text { Min Var cost and fuel cost (SA)( no } \\
\text { CA) }\end{array}$ & [25] [26] & \\
\hline $\begin{array}{l}\text { Min Var cost+ load shedding cost+ } \\
\text { preventive control cost } \\
\text { (MINLP)( GA/SLP)(CA) } \\
\text { (P-margin) }\end{array}$ & [27] & \\
\hline
\end{tabular}

Note: CA- contingency analysis

IPFLOW, VSTAB- software package for voltage stability analysis

PSAT - Power System Analysis Toolbox software

NLP - Nonlinear Programming

MINLP - Mixed Integer Nonlinear Programming

\section{REFERENCES}

[1] A. Calvaer, "Voltage stability and voltage collapse," CIGRE report, Paper 38.02, 1985.

[2] A. M. Chebbo, M. R. Irving, and M. J. H. Sterling, "Reactive power dispatch incorporating voltage stability," IEE Proc., vol. 139, no. 3, May 1992, pp. 253-260.

[3] US-Canada Power System Outage Task Force, Final Report on the August 14, 2003 Blackout in the United States and Canada: Causes and Recommendations.

[4] L. M. Tolbert, T. J. King, et al., "Power electronics etrategy for transmission and distribution and for distributed energy," ORNL /TM2005/230, Oak Ridge National Laboratory, Dec. 2005.
[5] G. Reed, J. Paserba, T. Croasdaile, el., "The VELCO STATCOMbased transmission system project," Proceedings of the 2001 IEEE PES Winter Power Meeting, Columbus, OH, vol. 3, 28 Jan.-1 Feb, 2001, pp. 1109 - 1114.

[6] G. Reed, J. Paserba, T. Croasdaile, el., "STATCOM application at VELCO Essex substation," Panel Session on FACTS Applications to Improve Power System Dynamic Performance, Proceedings of the IEEE PES T\&D Conference and Exposition, Atlanta, Georgia, vol. 2, 28 Oct.-2 Nov., 2001, pp. $1133-1138$.

[7] G. Reed, J. Paserba, T. Croasdaile, el., "SDG\&E Talega STATCOM project - system analysis, design, and configuration," Panel session on FACTS technologies: Experiences of the past decade and developments for the 21st century in Asia and the world, Proceedings of the IEEE PES T\&D-Asia Conference and Exposition, Yokahama, Japan, October 2002 .

[8] A. Scarfone, B. Oberlin, J. Di Luca Jr., D. Hanson, C. Horwill, M. Allen, "Dynamic performance studies for a \pm 150 Mvar STATCOM for Northeast utilities," Panel session on FACTS applications to improve power system dynamic performance, Proceedings of the IEEE PES T\&D Conference and Exposition, Dallas, Texas, Sep. 2003.

[9] W. Zhang, L. M. Tolbert, "Survey of reactive power planning methods," IEEE Power Engineering Society General Meeting, vol. 2, June 2005, San Francisco, California, pp. 1580-1590.

[10] C. Cañizares, "Calculating optimal system parameters to maximize the distance to saddle-node bifurcations," IEEE Trans. on Circuits and Systems -I: Fundamental Theory and Applications, vol. 45, no. 3, Mar. 1998, pp. 225-237.

[11] R. Ramos, J. Vallejos, B. Barán, "Multi-objective reactive power compensation with voltage security," 2004 IEEE/PES Transmission and Distribution Conference and Exposition: Latin America, Nov. 2004, Brazil, pp. $302-307$.

[12] V. Ajjarapu, P. L. Lau, and S. Battula, "An optimal reactive power planning strategy against voltage collapse," IEEE Transactions on Power Systems, vol. 9, no. 2, May 1994, pp. 906-917.

[13] H. Song, B. Lee, S. H. Kwon, V. Ajjarapu, "Reactive reserve-based contingency constrained optimal power flow (RCCOPF) for enhancement of voltage stability margins," IEEE Transactions on Power Systems, vol. 18 , no. 4, Nov. 2003, pp. 1538 - 1546.

[14] C. A. Cañizares, "Applications of optimization to voltage collapse analysis," Panel Session: Optimization Techniques in Voltage Collapse Analysis, IEEE-PES Summer Meeting, San Diego, California, July 1998

[15] C. A. Cañizares and S. Hranilovic, "Transcritical and Hopf bifurcations in ac/dc systems," Proc. Bulk Power System Voltage Phenomena IIIVoltage Stability and Security, Fairfax, VA, Aug. 1994, pp. 105-114.

[16] Y. Mansour, W. Xu, F. Alvarado, and C. Rinzin, "SVC placement using critical modes of voltage instability," IEEE Trans. on Power Systems, vol. 9, no. 2, May 1994, pp. 757-763.

[17] L. A. S. Pilotto, W. W. Ping, A. R. Carvalho, A. Wey, W. F. Long, C. L. DeMarco, F. L. Alvarado, A. Edris, "Determination of needed FACTS controllers that increase asset utilization of power systems," IEEE Transactions on Power Delivery, vol. 12, no. 1, Jan. 1997, pp. 363-371.

[18] M. A. Perez, A. R. Messina, C. R. Fuerte-Esquivel, "Application of FACTS devices to improve steady state voltage stability," 2000 IEEE Power Engineering Society Summer Meeting, vol. 2, July 2000, pp. $1115-1120$.

[19] M. K. Verma, S. C. Srivastava, "Optimal placement of SVC for static and dynamic voltage security enhancement," International Journal of Emerging Electric Power Systems, vol. 2, no. 2, 2005, Article 1050.

[20] C. S. Chang, J. S. Huang, "Optimal SVC placement for voltage stability reinforcement," Electric Power Systems Research, vol. 42, no. 3, Sep. 1997, pp. 165-172.

[21] C. S. Chang and J. S. Huang, "Optimal multiobjective SVC planning for voltage stability enhancement," IEE Proceedings- Generation, Transmission and Distribution, vol. 145, no. 2, Mar. 1998, pp. 203 209.

[22] H. Mori, H. Tani, "Two-staged tabu search for determining optimal allocation of D-FACTS in radial distribution systems with distributed generation," 2002: Asia Pacific IEEE/PES Transmission and Distribution Conference and Exhibition, vol. 1, Oct. 2002 , pp. $56-61$.

[23] L. J. Cai, I. Erlich, G. Stamtsis, "Optimal choice and allocation of FACTS devices in deregulated electricity market using genetic algorithms," 2004 IEEE/PES Power Systems Conference and Exposition, vol. 1, Oct. 2004, pp. 201 - 207. 
[24] K. Habur, and D. Oleary, "FACTS - flexible AC transmission systems, for cost effective and reliable transmission of electrical energy," http://www.siemenstd.com/TransSys/pdf/CostEffectiveReliabTrans.pdf.

[25] L. J. Cai, I. Erlich, "Optimal choice and allocation of FACTS devices using genetic algorithms," ISAP, Intelligent Systems Application to Power Systems, 2003, Lemnos, Greece, Aug. 31 -Sep. 3, 2003.

[26] R. Palma-Behnke, L. S. Vargas, J. R. Pérez, J. D. Núñez, R. A. Torres, "OPF with SVC and UPFC modeling for longitudinal systems," IEEE. Trans. Power Systems, vol. 19, no. 4, Nov. 2004, pp. 1742-1753.

[27] E. E. El-Araby, N. Yorino, H. Sasaki, "A comprehensive approach for FACTS devices optimal allocation to mitigate voltage collapse," Proc. of IEEE/PES Transmission and Distribution Conference, vol. 1, Oct. 2002, pp. $62-67$.

[28] P. Preedavichit and S. C. Srivastava, "Optimal reactive power dispatch considering FACTS devices," Proceedings of the $4^{\text {th }}$ International Conference on Advances in Power System Control, Operation and Management, APSCOM-97, Hong Kong, Nov. 1997, pp. 620-625.

[29] P. Bhasaputra, W. Ongsakul, "Optimal power flow with multi-type of FACTS devices by hybrid TS/SA approach," 2002 IEEE International Conference on Industrial Technology, vol. 1, Dec. 2002, pp. 285 - 290.

[30] R. Adapa, K. Madajewski, M. Sobierajski, "The study of voltage problems in Polish power system," 2000 IEEE Power Engineering Society Winter Meeting, vol. 3, pp. 1727-1732.

[31] R. Natesan, G. Radman, "Effects of STATCOM, SSSC and UPFC on voltage stability," 2004 Proceedings of the Thirty-Sixth Southeastern Symposium on System Theory, 2004, pp. 546 - 550.

[32] H. Yonezawa, T. Shimato, M. Tsukada, K. Matsuno, I. Iyoda, J. J. Paserba, G. F. Reed, "Study of a STATCOM application for voltage stability evaluated by dynamic PV curves and time simulation," 2000 IEEE Power Engineering Society Winter Meeting, vol. 2, Jan. 2000, pp. $1471-1476$

[33] H. G. Sarmiento, G. Pampin, J. D. de Leon, "FACTS solutions for voltage stability problems in a large metropolitan area," 2004 IEEE/PES Power Systems Conference and Exposition, vol. 1, Oct. 2004, pp. $275-282$.

[34] G. K. Morison, B. Gao, P. Kundur, "Voltage stability analysis using static and dynamic approaches," IEEE Transactions on Power Systems, vol. 8, no. 3, Aug. 1993, pp. $1159-1171$.

\section{BIOGRAPHIES}

Wenjuan Zhang (S' 2003) is presently a Ph.D. candidate at The University of Tennessee. She received the B.S.E.E. from Hebei University of Technology, China, in 1999 and the M.S.E.E. from Huazhong University of Science and Technology, China, in 2003. Her current interests include reactive power compensation and planning, voltage stability, optimization.

Fangxing (Fran) Li (M' 2001, SM'2005) has been an Assistant Professor in the ECE Department at The University of Tennessee (UT), Knoxville, TN since August 2005. His current interests include reactive power, distributed generation, energy markets, reliability, computational methods, and power electronics. He received his Ph.D. degree from Virginia Tech in 2001. Dr. Li is the recipient of the 2006 Eta Kappa Nu Outstanding Teacher Award at UT.

Leon M. Tolbert (SM' 1999) received his B.S., M.S., and Ph.D. degrees all from Georgia Tech, Atlanta in 1989, 1991, and 1999, respectively. He joined the Engineering Division of Oak Ridge National Laboratory (ORNL) in 1991. He was appointed as an assistant professor in the ECE Department at The University of Tennessee in Knoxville in 1999. He is presently an Associate Professor at UT and a research engineer at ORNL. He is a Registered Professional Engineer in the state of Tennessee and the Chairman of the Education Activities Committee of the IEEE Power Electronics Society. 